\title{
Attenuation of Paraquat-Induced Dopaminergic Toxicity on the Substantia Nigra by (-)-Deprenyl in Vivo
}

\author{
Horng-Huei Liou, ${ }^{*} \uparrow^{, 1}$ Rong-Chi Chen, $\dagger \dagger$ Tony Hsiu-Hsi Chen, $\S$ Yuan-Feen Tsai, ${ }^{\mathbb{I}}$ and Ming-Cheng Tsai* \\ Departments of *Pharmacology, †Neurology, and "Physiology, College of Medicine, and §Institute of Epidemiology, College of Public Health, \\ National Taiwan University, Taipei, Taiwan; and $\ddagger$ Department of Neurology, En Chu Kong Hospital, Taipei, Taiwan
}

Received September 13, 2000; accepted December 21, 2000

\begin{abstract}
Attenuation of Paraquat-Induced D opaminergic T oxicity on the Substantia Nigra by (-)-Deprenyl in Vivo. Liou, H.-H., Chen, R .-C ., C hen, T. H .-H ., T sai, Y .-F ., and T sai, M.-C . (2001). Toxicol. Appl. Pharmacol. 172, 37- 43.
\end{abstract}

(-)-Deprenyl (DEP) had been shown to slow of progression of Parkinson's disease (PD). The present study sought to determine whether DEP would attenuate the nigrostriatal system damage induced by intranigral administration of the herbicide paraquat $(P Q)$ as a model of parkinsonism in vivo. Neurochemical and behavioral observations of $W$ istar rats were the focus of our study. In the neurochemical observation, the $P Q$ injected in the rats caused dose-dependent depletion of dopamine (DA) in the ipsilateral striata. The coadministration of DEP with $P Q$ partially increased the striatal DA level. The prediction of the striatal DA levels was calculated by regression coefficients obtained from multiple linear regression $\left(r^{2}=0.82\right)$ : DA level ( $\%$ of control) $=$ 103.34 - 9.58 PQ (nmol) + 0.79 DE P (nmol). It was demonstrated that the high dose of $20 \mathrm{nmol} D E P$ could significant attenuate the PQ $(5 \mathrm{nmol})$-elicited dopaminergic toxicity $(p<0.05)$. In the behavioral observation, the intranigral injection of $P Q$ into the rats caused a rotation behavior contralateral to the lesioned side in response to apomorphine administration $(0.5 \mathrm{mg} / \mathrm{kg}, \mathrm{sc})$. This apomorphine-induced rotational behavior could also be attenuated significantly by coadministration of D E P $(20 \mathrm{nmol})$ and PQ (5 nmol) compared with PQ-treated $(5 \mathrm{nmol})$ animals $(p<0.05)$. The above observations indicate that DEP could provide a protective effect on the moderate injury elicited by $P Q$ toxicity of the nigrostrital dopaminergic system. DEP might be a useful therapeutic agent in treating patients with early-stage PD. $\odot 2001$ Academic Press

Key Words: paraquat; deprenyl; dopamine; Parkinson's disease.

Progressive loss of nigrostriatal dopaminergic (DAergic) neurons may elicit Parkinson's disease (PD). Increasing evidence supports the hypothesis that environmental factors contribute to PD development (for review, see Tanner, 1989). The discovery that 1-methyl-4-phenyl-1,2,3,6-tetrahydropyridine (MPTP) administration results in a syndrome resembling the clinical, biochemical, and pathological features of PD in hu-

${ }^{1}$ To whom correspondence should be addressed. Fax: 011-886-2-23418395. E-mail: hhliou@ha.mc.ntu.edu.tw. mans and experimental animals has stimulated a search for the environmental chemicals resembling MPTP that might cause PD (Langston et al., 1983; Burns et al., 1983; Ballard et al., 1985). MPTP is converted by the action of monoamine oxidase $\mathrm{B}$ (MAO-B) to 1-methyl-4-phenylpyridinium ion $\left(\mathrm{MPP}^{+}\right)$, the effective neurotoxin (Javitch et al., 1985; Singh et al., 1988). Paraquat $\left(1,1^{\prime}\right.$,-dimethyl-4,4'-bipyridium dichloride; PQ) is a widely used herbicide. Because of the striking similarity in structure between PQ and $\mathrm{MPP}^{+}, \mathrm{PQ}$ may cause toxic effects to the DA neurons as $\mathrm{MPP}^{+}$. From the epidemiological observation, a strong correlation has been reported between the incidence of PD and the level of PQ (Barbeau et al., 1986; Ho et al., 1989; Hertzman et al., 1990; Tanner and Goldman, 1996; Liou et al., 1997). PQ has been shown to induce nigrostriatal neuronal degeneration in rodents (Endo et al., 1988; Fredriksson et al., 1993; Liou et al., 1996; Brooks et al., 1999). These findings suggest that PQ might play an important role in the pathogenesis of PD.

The mechanism of PQ has been suggested to be mediated by a series of free radical reactions (Bus et al., 1976; Trush et al., 1981, Kadiiska et al., 1993). These highly toxic radicals are extremely reactive with macromolecules and may result in multiple organ injuries, leading to death in several species. Recognizing the fact that PQ is a strong redox agent and contributes to the formation of reactive oxygen species (ROS), attempts have been made to explore pharmacological strategies that may reduce the formation of these ROS and/or prevent their toxic effects. Intranigral infusion of $\mathrm{Cu}$-free superoxide dismutase (SOD) prevents PQ-induced behavioral stimulation and electrocortical epileptogenic discharges in rats (Iannone et al., 1991). Melatonin protects against PQ-induced toxicity and genotoxicity is mediated by its free radical scavenging activity (Melchiorri et al., 1996, 1998).

(-)-Deprenyl (selegiline; DEP), a selective type B monoamine oxidase inhibitor, is an adjuvant to levodopa therapy of PD. DEP has been shown to delay the emergence of disability and the progression of signs and symptoms in the early phase of the disease (Parkinson Study Group, 1993). In addition to its antiparkinsonian actions, DEP has prevented the neurotoxicity of MPTP in monkeys (Cohen et al., 1984) and has protective 
effects against toxin-induced neuronal deterioration (Finnegan et al., 1990; Wu et al., 1993, 1996; Salonen et al., 1996). DEP has been reported to reduce the toxic effect of $\mathrm{MPP}^{+}$via its antioxidant effect in vivo (Wu et al., 1993, 1996). Low doses of DEP administration after the insult have been shown to possess neuronal rescue-like properties with an unknown mechanism, possibly independent of MAO-B inhibition, in different degenerative models both in vitro and in vivo (Ansari et al., 1993; Wu et al., 1993; Ju et al., 1994; Tang et al., 1998). Furthermore, DEP has shown antiapoptotic properties in vitro (Tatton et al., 1994; Wadia et al., 1998; Carlile et al., 2000). DEP is thus considered to be a potential effective neuroprotective agent.

Both $\mathrm{MPP}^{+}$and PQ have similar effects on free radical generation (Johannessen et al., 1986; Fallon et al., 1997; Lotharius et al., 1999) and could induce nigrostriatal degeneration. However, The effect of DEP on PQ-elicited nigrostriatal dopaminergic toxicity in vivo remains unclear. The primary goal of this study was to examine the possibility of whether DEP could attenuate nigrostriatal system damage by the intranigral administration of the herbicide PQ. Drug-induced behavioral asymmetries observed on the tested animals with unilateral destruction of the nigrostriatal DA system were used as an experimental model to study PD (Ungerstedt and Arbuthnott, 1970) and to evaluate the recovery of DA neuronal function by therapeutic approaches (Perlow et al., 1979; Wang et al., 1995; Hoffman et al., 1997). The present study was performed by direct intracerebral injection of PQ and DEP into the unilateral substantia nigra compacta $(\mathrm{SNc})$ of the rats. The effects of DEP on PQ-induced toxicity in nigrostriatal system were assessed by striatal neurochemistry and behavioral observations in vivo.

\section{MATERIALS AND METHODS}

Animals. Male Wistar rats (3 months old) were housed 4 per cage in a temperature-regulated room $\left(23 \pm 2^{\circ} \mathrm{C}\right)$ and maintained on a $12: 12 \mathrm{~h}$ light: dark cycle (lights on at $0600 \mathrm{~h}$ ), with food and water available ad libitum. The animals were acclimated for at least one week before the surgical procedure. The rats weighed 250-300 g at the time of the stereotaxic operation.

Stereotaxic operation. The rats were anesthetized by pentobarbital sodium (30 mg/kg, ip) and mounted in a stereotaxic apparatus (David Kopf instruments). A 2-cm long incision was made in the midline of scalp to expose the skull and a 0.5 -mm-diameter hole was drilled in the calvaria over the right $\mathrm{SNc}$ for the insertion of a 30-gauge injection needle by using the Paxinos and Waston (1986) coordinates: AP 5.3, L 2.0, H $7.8 \mathrm{~mm}$. All rats underwent intranigral injection; they were divided into 4 major groups with different drugs administration protocol. Group A received intranigral injection of PQ $(2.5,5$, and 10 nmole, respectively, $n=7$ in each group). Group B received intranigral co-administration of PQ and DEP. They were administrated with the following listed dosage regimen of PQ and DEP (nmole): PQ $2.5+$ DEP 5, PQ $2.5+$ DEP 20, PQ $5+$ DEP 5, PQ $5+$ DEP 20, PQ $10+$ DEP 5, and PQ $10+$ DEP 20, respectively ( $n=7$ in each group). Group $\mathrm{C}$ received intranigral injection of DEP 5 and 20 nmole, respectively $(n=7$ in each group). Group D, the sham-operated control animals, received intranigral injection of saline $(n=7)$. PQ (Sigma, St. Louis, MO, USA) and DEP (RBI, Natick, MA, USA) were dissolved in normal saline and were slowly infused
$(0.2 \mu \mathrm{l} / \mathrm{min})$ by an infusion pump through a 30 -gauge stainless steel needle into the right SNc. The injection needle was left in position for a further $2 \mathrm{~min}$ following the infusion of total $1 \mu \mathrm{l}$ of drug solution. The position of the injection site was verified histologically at postmortem.

Striatal dopamine level assayed by HPLC. Animals were scarified by decapitation two weeks after intranigral injection of substance. The brains were quickly removed and immediately frozen in isopentane at $-20^{\circ} \mathrm{C}$. Serial cryostat sections (200 $\mu \mathrm{m}$ thick) were cut in the frontal plane, the tissues of the striatum micropunched (Palkovits, 1973), and the micropunched tissues homogenized in perchloric acid $(0.1 \mathrm{M})$, using an ultrasonic cell disruptor (Heat System Ultrasonic, USA), then centrifuged at $3000 \mathrm{~g}$ for $3 \mathrm{~min}$. The supernatants were removed and assayed for DA, 3,4-dihydroxyphenylacetic acid (DOPAC), and homovanillic acid (HVA) using high-pressure liquid chromatography (HPLC) with electrochemical detection. A C-18 reverse phase column ( $5 \mu \mathrm{m}, 12.5 \mathrm{~cm}$ long, Waters Chromatography Division, USA) was connected to a carbon electrode set at a potential of $0.75 \mathrm{~V}$ relative to the $\mathrm{Ag} / \mathrm{AgCl}$ reference electrode, together with an LC-4 amperometric detector (Bioanalytic System Inc., USA) in the HPLC system (Tsai et al., 1994; Liou et al., 1996).

Each liter of the mobile phase used in the experiment contained heptanesulfonic acid (1.75 g), disodium EDTA (0.1 g), triethylamine $(3.5 \mathrm{ml})$, phosphoric acid (4 ml) and acetonitrile (40 ml) in distilled water (Tsai et al., 1994; Liou et al., 1996). It was filtered and degassed just prior to use. External standards of DA, DOPAC and HVA were dissolved in perchloric acid $(0.1 \mathrm{M})$ and run at the same time as the experimental samples. The protein contents were assayed from tissue pellets solubilized in $\mathrm{NaOH}(0.5 \mathrm{M})$ by the method of Lowry et al. (1951).

Behavioral observations and pharmacological tests. The spontaneous behavior of rats was observed after unilateral intranigral injection of substances, when left undisturbed in their cages or when disturbed by handling, pinching of their tails, and sudden noise. Apomorphine-induced rotational behavior was assessed utilizing a computerized rotometer system (Hudson et al., 1993) described by Ungerstedt and Arbuthnott (1970). Rats were tested 10 days after intranigral injection of the test substance or saline. The rats were placed in rotometer bowls and secured to the counting head by a thoracic harness. After acclimation for at least $10 \mathrm{~min}$, an injection of apomorphine (Sigma, St. Louis, MO, USA, $0.5 \mathrm{mg} / \mathrm{kg}, s c$ ) was administered. Animals that had completed a $360^{\circ}$ circle towards the intact (contralateral) side and/or the lesion (ipsilateral) side were monitored and recorded by computer every minute for $2 \mathrm{~h}$ continuously (Hudson et al., 1993; Wang et al., 1995).

Statistical methods. All observed values are expressed as the mean $\pm \mathrm{SE}$. The overall analyses for comparison of mean values were performed by one-way analysis of variance (ANOVA). Statistical significant level ( $\alpha$ ) was set up at 0.05 . Post-hoc tests (i.e., Scheffe's method) were used to examine the specific difference between any of two groups while the results of the overall analysis reach statistical significance. The relationship of DA to PQ and DEP was analyzed by the use of multiple linear regression. The association between the rotational behavior and striatal DA levels was analyzed by simple linear regression. The strength of this relationship was quantified by square of correlation coefficient $\left(r^{2}\right)$.

\section{RESULTS}

\section{Striatal Dopamine Content Assayed by HPLC}

The injection of PQ into the SNc resulted in reducing the striatal DA levels. The effect depended on the dose of PQ injected. Following intranigral administration of PQ $(2.5,5$, and $10 \mathrm{nmole}$ ), the DA levels in the ipsilateral striata were decreased to $81.6,47.9$ and $13.5 \%$ of saline-treated control. PQ (5 and 10 nmole) significantly decreased the striatal DA levels compared with controls $(p<0.05)$. 


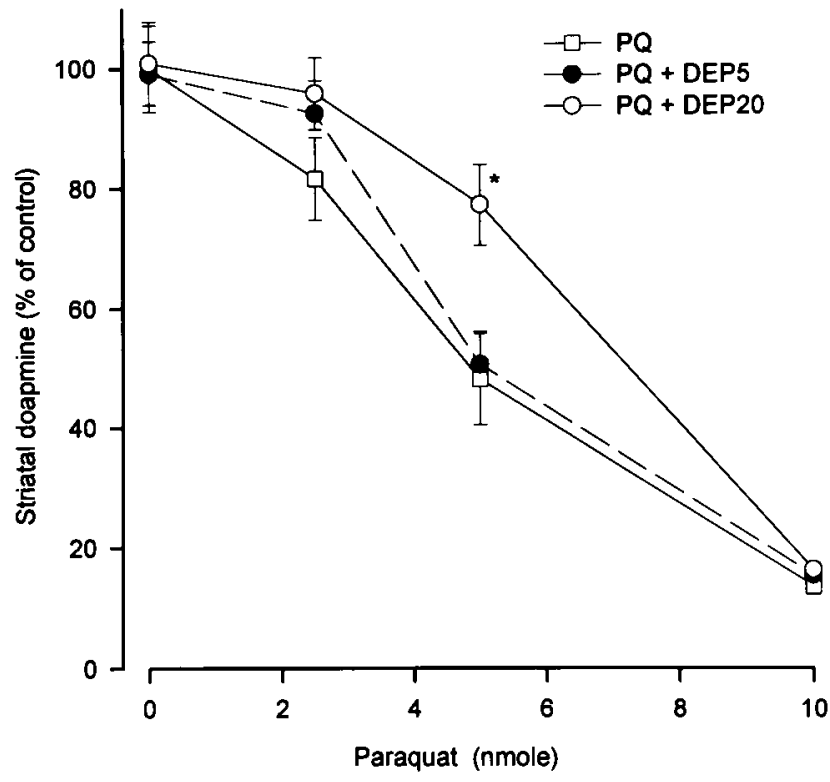

FIG. 1. Effects of unilateral intranigral administration of (-)-deprenyl (DEP) and/or paraquat (PQ) on dopamine (DA) contents of the ipsilateral striatum. Animals were scarified two weeks after treatment $(n=7$, in each dosage regimen). The striata were micropunched and assayed for DA level by HPLC. The striatal DA contents were expressed as percentage of controls. It was notable that the animals which co-administrated DEP (20 nmole) and PQ (5 nmole) were significantly increased in the striatal DA levels compared with those of PQ (5 nmloe) $(p<0.05)$. PQ + DEP 5: co-administration of PQ and 5 nmole DEP, PQ + DEP 20: coadministration of PQ and 20 nmole DEP.

The effects of DEP on PQ-elicited striatal DA toxicity were showed in Fig. 1. Co-administration of low dose of PQ (2.5 nmole) and DEP (5 and 20 nmole) into SNc attenuated the PQ induced toxicity manifested as the ipsilateral striatal DA levels to be increased to $92.4 \%$ and $95.8 \%$ of control. In the animals which received PQ (5 nmole) elicited moderate striatal DA toxicity $(47.9 \%)$, co-administration of PQ (5 nmole) and DEP (5 and 20 nmole) increased the DA levels to $50.4 \%$ and $77.2 \%$ of control. Co-administration of PQ (10 nmole) and DEP (5 and 20 nmole) increased the striatal DA contents to $16.5 \%$ and $16.2 \%$ of control. Results of ANOVA showed only the effect of DEP on PQ (5 nmole) on DA level reach statistic significance $\left(\mathrm{F}_{(2,18)}=5.84, p=0.01\right)$. Subsequent Scheffe's post-hoc assessment showed that the DA levels in the group of coadministration of DEP (20 nmole) and PQ (5 nmole) were significant higher than those in PQ $(5$ nmole $)$ and co-administration of PQ ( 5 nmole) and DEP $(5$ nmole) $(p<0.05)$. PQ $(5$ nmole) elicited moderated DA toxicity was significantly attenuated by co-administration of high dose of DEP (20 nmole). When the striatal DA level depleted more than $85 \%$ by PQ (10 nmole), co-administration DEP (5 and 20 nmole) could not attenuate the PQ elicited severe striatal DA toxicity in rats.

We further analyzed the dose-response effects between the PQ and DEP in the striatal DA level. The prediction of striatal DA levels was calculated by regression coefficients obtained from multiple linear regression $\left(r^{2}=0.82\right)$ : striatal DA level $(\%$ of control $)=103.34-9.58 \mathrm{PQ}($ nmole $)+0.79 \mathrm{DEP}$ (nmole).

Table 1 showed the effects of DEP on PQ-treated animals on levels of striatal DA and its metabolites. It was demonstrated that DEP in various doses led to a recovery of DA, DOPAC and HVA in the neostriatum. The PQ-induced increase in DA utilization as seen from the (DOPAC+HVA)/DA ratio was not counteracted by the DEP treatment.

\section{Behavioral Studies}

Figure 2 showed the apomorphine $(0.5 \mathrm{mg} / \mathrm{kg}, s c)$ induced rotational behavior in rats 10 days after unilateral intranigral

TABLE 1

E ffects of (-)-D eprenyl on Paraquat-T reated Animals on Levels of Striatal Dopamine and Its Metabolites

\begin{tabular}{|c|c|c|c|c|c|}
\hline & DOPAC $(\mathrm{pg} / \mu \mathrm{g})$ & $\mathrm{DA}(\mathrm{pg} / \mu \mathrm{g})$ & 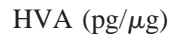 & DOPAC/DA & HVA/DA \\
\hline Control & $27.94 \pm 2.58$ & $184.64 \pm 13.26$ & $9.24 \pm 1.12$ & $0.14 \pm 0.01$ & $0.05 \pm 0.01$ \\
\hline DEP (5) & $28.69 \pm 2.72$ & $182.72 \pm 10.37$ & $10.05 \pm 1.67$ & $0.14 \pm 0.01$ & $0.05 \pm 0.01$ \\
\hline DEP (20) & $31.29 \pm 4.36$ & $186.26 \pm 12.79$ & $11.51 \pm 1.70$ & $0.13 \pm 0.01$ & $0.05 \pm 0.01$ \\
\hline PQ (2.5) & $24.42 \pm 3.15$ & $150.62 \pm 12.67$ & $6.38 \pm 1.27$ & $0.16 \pm 0.01$ & $0.04 \pm 0.01$ \\
\hline PQ (2.5) + DEP (5) & $22.04 \pm 2.59$ & $170.69 \pm 10.04$ & $7.05 \pm 1.71$ & $0.13 \pm 0.01$ & $0.04 \pm 0.01$ \\
\hline PQ $(2.5)+$ DEP (20) & $23.08 \pm 4.21$ & $176.84 \pm 11.02$ & $7.21 \pm 1.26$ & $0.13 \pm 0.01$ & $0.05 \pm 0.01$ \\
\hline $\mathrm{PQ}(5)$ & $20.17 \pm 2.51$ & $88.62 \pm 14.17$ & $5.14 \pm 0.94$ & $0.22 \pm 0.04$ & $0.06 \pm 0.01$ \\
\hline PQ (5) + DEP (5) & $20.08 \pm 3.01$ & $93.12 \pm 10.21$ & $5.09 \pm 0.76$ & $0.21 \pm 0.03$ & $0.06 \pm 0.01$ \\
\hline PQ (5) + DEP (20) & $25.26 \pm 3.28^{*}$ & $142.48 \pm 12.39 *$ & $6.20 \pm 0.81^{*}$ & $0.19 \pm 0.02$ & $0.05 \pm 0.01$ \\
\hline PQ (10) & $6.94 \pm 1.24$ & $24.84 \pm 2.76$ & $2.70 \pm 0.24$ & $0.25 \pm 0.04$ & $0.11 \pm 0.01$ \\
\hline PQ (10) + DEP (5) & $7.32 \pm 1.84$ & $30.19 \pm 2.41$ & $2.19 \pm 0.76$ & $0.24 \pm 0.04$ & $0.10 \pm 0.01$ \\
\hline PQ (10) + DEP (20) & $7.20 \pm 1.64$ & $29.92 \pm 2.10$ & $2.41 \pm 0.92$ & $0.24 \pm 0.03$ & $0.08 \pm 0.01$ \\
\hline
\end{tabular}

Note. Concentrations (in pg/ $\mu \mathrm{g}$ protein) of dopamine (DA), 3,4-dihydroxyphenylacetic acid (DOPAC), and homovanillic acid (HVA) in the striatum 2 weeks after unilateral intranigral injection of (-)-deprenyl (DEP) and/or paraquat (PQ) were listed. Parentheses show the concentration (nmole) of injected PQ and/or DEP. Values are expressed as the mean \pm SE for each group $(n=7)$.

* Statistically significant difference compared with the same dose of PQ-treated animals and the coadministration with DEP and PQ-treated rats $(p<0.05)$. 


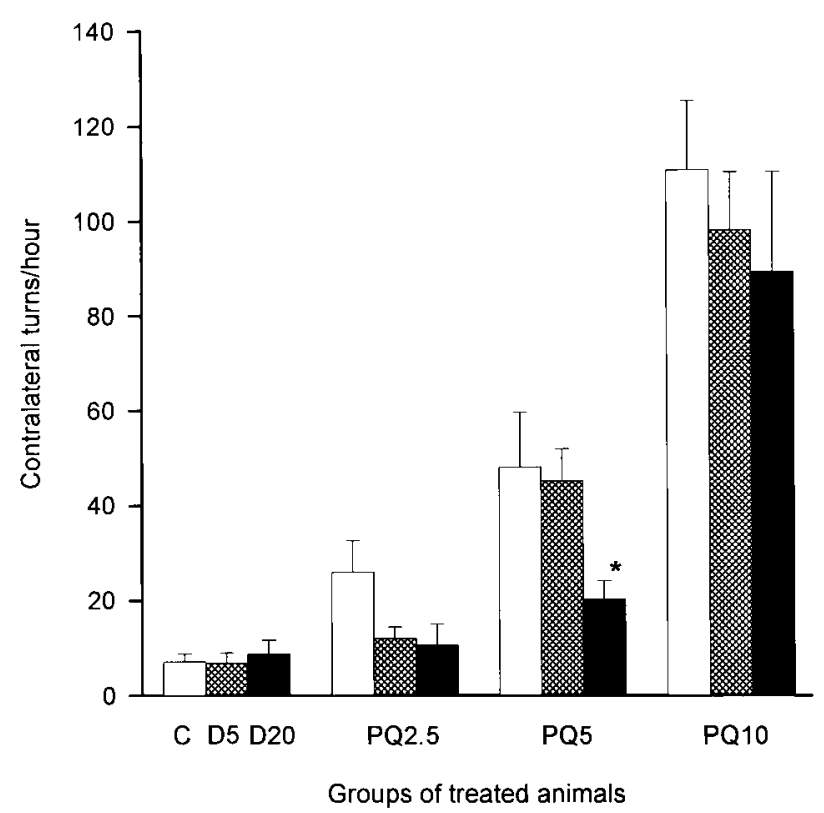

FIG. 2. Effects of apomorphine $(0.5 \mathrm{mg} / \mathrm{kg}, s c)$ on contralateral rotational behavior in rats 10 days after unilateral intranigral injection of (-)-deprenyl (DEP) and/or paraquat $(\mathrm{PQ})$. Each column showed the mean \pm SE from 7 rats in each group of treatment. At groups C, D5, and D20, the rats received intranigral injection of saline (control), DEP 5 nmole, and 20 nmole, respectively. At PQ 2.5, PQ 5, and PQ 10, the rats received intranigral administration of PQ 2.5 nmole, 5 nmole, and 10 nmole with or without co-administration of DEP. White columns represented the rats which did not co-administrate with DEP. The hatch columns represented the rats which co-administrated with DEP 5 nmole, and the black columns represented the rats which co-administrated DEP 20 nmole. The statistical significant difference in the numbers of contralateral turning per hour compared with the same dose of PQ- and DEP co-administration with PQ-treated group were as shown, $p<0.05\left(^{*}\right)$. Noted that DEP (20 nmole) co-administration with PQ (5 nmole) significantly attenuated the PQ-elicited $(5 \mathrm{nmole})$ rotational behavioral after apomorphine treatment.

injection of DEP and/or PQ. Apomorphine induced rare contralateral rotational behavior in the saline-treated controls $(7 \pm$ 2 turns/h, $n=7$ ) and DEP-treated ( 5 and 20 nmole) rats ( $7 \pm$ 2 and $9 \pm 4$ turns/h, $n=7)$. In rats receiving intranigral injection of PQ (2.5, 5 and 10 nmole), the apomorphine elicited contralateral rotational behavior at a rate of $26 \pm 8,48 \pm 12$, and $110 \pm 14$ turns/h $(n=7$ in each dosage), which was statistically significant compared with saline- and DEP-treated animals $(p<0.05)$.

Co-administration of PQ and DEP into SNc could attenuate the apomorphine induced contralateral rotational behavior in rats treated with PQ dose-dependently. Apomorphine induced contralateral rotational behavior in the rats that received coadministrated low dose of PQ (2.5 nmole) and DEP (5 and 20 nmole) at the rates of $12 \pm 2$ and $11 \pm 5(n=7)$. In the rats injected with the moderate dose of PQ ( 5 nmole) and DEP (5 and $20 \mathrm{nmole}$ ), apomorphine elicited contralateral rotational behavior in rats at the rates of $45 \pm 7$ and $20 \pm 4(n=7)$. Likewise, apomorphine elicited rotational behavior in the rats treated with high dose of PQ (10 nmole) and DEP (5 and 20 nmole) at the rates of $97 \pm 12$ and $89 \pm 23(n=7)$. Among these treated rats, co-administration of DEP (20 nmole) and PQ (5 nmole) revealed significantly that decreased apomorphine elicited rotational behavior compared with those rats that received PQ $(5$ nmole $)(p<0.05)$.

There is a well correlation between the change in striatal DA levels and behavior measure of rotation using individual animal data $\left(r^{2}=0.73\right)$. A simple linear regression equation for the relationship between rotational behavior and striatal DA levels was: animal total contralateral turns $/ \mathrm{h}=-0.56$ (striatal DA levels) +108.26 .

\section{DISCUSSION}

The present study demonstrated that DEP could attenuate the toxic effects elicited by PQ on striatal DA and its metabolites. In accordance with neurochemical findings, DEP caused significant diminution of apomorphine-induced rotational behavior in rats whose striata had been unilateral lesioned with PQ. These neurochemical and behavioral results suggest that DEP could protect nigrostriatal neurons from PQ-induced DA-ergic toxicity.

PQ has been extensively studied as a both a pulmonary and neurotoxicant. Unlike the accidental and high-level PQ exposure that produced acute pulmonary toxicity, it was presumed that the chronic low-level nonpulmonary toxic doses could produce a different syndrome defined by damage to basal ganglia and parkinsonism. Little is actually known about actual human exposure levels to PQ and the routes by which they occur, although it is likely that they would include inhalation, per oral ingestion or through transdermal absorption. Low levels of PQ appear to be retained in tissue such as muscle after subcutaneous exposures from where it can then be slowly released into blood (Sharp et al., 1972). Several investigators suggest PQ was a causal factor for PD (Barbeau et al., 1986; Ho et al., 1989; Hertzman et al., 1990; Tanner and Goldman, 1996; Liou et al., 1997). Bocchetta and Corsini (1986) reported two patients believed to suffer from PQ-induced parkinsonism. Sanchez-Ramos et al. (1987) reported a young farmer who had been exposed to PQ and affected with PD. Animal studies also showed that repeated systemic injection of PQ was sufficient to reduce locomotor activity and the decline of DA-ergic neurons in mice (Brooks et al., 1999). Systemical administration of $\left[{ }^{14} \mathrm{C}\right] \mathrm{PQ}$ indicated that the herbicide did partition in the CNS presumably by penetration of the endothelium comprising the blood-brain barrier (Lindquist et al., 1988). The autoradiographic studies with systemically applied $\left[{ }^{14} \mathrm{C}\right] \mathrm{PQ}$ also demonstrated that the compound was most highly confined to neuromelanin producing cells such as those in the $\mathrm{SNc}$ (Lindquist et al., 1988). Such a pattern of distribution suggests that PQ can preferentially enter and/or be maintained in cells which elaborate neuromelanin.

PQ possesses marked neurotoxicity for the nigro-stritatal DA-ergic system (Endo et al., 1988; Fredriksson et al., 1993; 
Liou et al.,1996; Yang and Sun, 1998; Brooks et al., 1999). We found that the intranigral administration of PQ caused a dosedependent decrease of DA in the major terminal area, the striatum. Similar pharmacological results were seen in MPP ${ }^{+}$ -treated rats (Bradbury et al., 1986; Sirinathsinghji et al., 1988; Sun et al., 1988). Our results revealed that DEP could provide protective effect against PQ-induced moderate nigral injury as reflected by a $50 \%$ or less depletion of DA in the striatum. As the dose of PQ elicited more than $85 \%$ depletion of striatal DA, DEP could not exert its protective effect. These observations were consistent with previous findings on the effect of DEP in $\mathrm{MPP}^{+}$-treated rats in vivo (Wu et al., 1993, 1996).

Unilateral lesions within the nigrostriatal DA-ergic system of the rats induced a rotational behavior, which reflected an imbalance of DA-ergic activity in the striata (Ungerstedt and Arbuthnott, 1970; Creese et al., 1977). Behavioral supersensitivity was manifested by the rats rotating in a direction contralateral to the side of the lesion following the systemic administration of a DA agonist, such as apomorphine, and appeared to be caused by supersensitivity of the denervated striatal DA receptors (Ungerstedt and Arbuthnott, 1970; Creese et al., 1977; Hudson et al., 1993). This behavior correlated with the extent of DA depletion and thus was utilized as a functional index for the recovery of DA-containing neurons following therapeutic interventions (Perlow et al., 1979; Wang et al., 1995; Hoffman et al., 1997). The present experiment showed that the apomorphine could induce a degree of vigorous contralateral rotational behavior in rats after unilateral intranigral injection of PQ in a dose-dependent manner. These rotational behaviors were significantly reduction by administration of DEP (20 nmole) in the PQ-treated ( 5 nmole) animals. This restored rotational behavior manifestation was similar to the fetal DA homografts in unilateral 6-hydroxy-dopamine (6-OHDA) lesioned rats (Wang et al., 1995).

The present study showed that apomorphine-induced contralateral circling behavior seen after unilateral intranigral injection of PQ correlated well with the decrease in striatial DA levels, and the neurochemical and behavioral changes induced by PQ were attenuated by administration of DEP. It suggested that the decrease in turning was related to the protective action of DEP on PQ-induced DA depletion.

The increases in the ratio of DA metabolites to DA indicated that the compensatory mechanisms could be aroused to increase the DA release in the brain of PD or in brain of the tested animals that had been treated with 6-OHDA, MPTP and PQ (Palkovits, 1973; Sirinathsinghji et al., 1988; Liou et al., 1996). The present experiment showed no change in the ratio of DA metabolites to DA between the PQ-treated and coadministrated with PQ and DEP-treated rats. These data indicated that DEP might not increase striatal DA levels via its metabolites such as methamphetamine or amphetamine (ThyagaRajan et al., 1999), which had been shown to enhance DA release or alter the DA turnover (reflected as the ratio of DOPAC/DA) by its inhibitory action of MAO-B activity.
Although the mechanisms of how DEP operated its effects on PQ were not known for certain, several lines of evidence indicated that DEP might exert its protective actions via its antioxidative properties. DEP had been reported to suppress the formation of free radicals from MPTP and its analogues (Chiueh et al., 1992; Wu et al., 1996) and it could scavenge hydroxyl and peroxyl radicals both in vivo and in vitro (Cohen and Spina, 1989; Wu et al., 1993, 1996; Thomas et al., 1997). Besides, DEP could prevent quinolinic acid-induced hippocampal damage by a mechanism of interfering ROS generation (Behan et al., 1999). In addition to the putative antioxidant properties, the neuroprotection of DEP had been considered to be associated with several intracellular mechanisms, including enhancement of antioxidant enzymes, such as SOD and catalase (Carrillo et al., 1992), inhibition of the uptake of DA (Zsilla et al., 1986), preservation of mitochondrial membrane potential (Wadia et al., 1998), activation of antiapopotic system (Tatton et al., 1994; Maruyama and Naoi, 1999; Carlile et al., 2000), and increase in mRNA of trophic factors, such as BDNF (Tang et al., 1998), NGF (Semkov et al., 1996) and CTNF (Seniuk et al., 1994). DEP might probably act like an antioxidant and/or scavenger, inhibiting the free radical formation and oxidative injury elicited by $\mathrm{PQ}$ in the nigrostriatal system in this experimental paradigm, but the exact mechanism remained to be elucidated.

$\mathrm{PQ}$ is a generator of superoxide anions which were known to induce cell damage, either directly by blocking cell respiratory chain (Patel et al., 1996), or indirectly by activation of cholinergic (Seto and Shinohara 1988) and glutamatergic (PellegriniGiampietro et al., 1990; Bagetta et al., 1992) transmission that causes neuronal death via an excitotoxic mechanism. Polyamine metabolism was a secondary target for PQ toxicity. PQ can interfere with polyamine synthesis and uptake, producing arrested growth and eventually cell death (Masek and Richards, 1990; Bayoumi et al., 2000). The intracellular spermidine and spermine pools were negatively affected with PQ in a dose-response manner (Bayoumi et al., 2000). Our results illustrated that DEP apparently had protective effect on rats which were given a moderate, but not high, dose of PQ elicited neurotoxicity. As to whether a high dose of PQ might possess more potent toxicity to generate mechanism that was beyond the neuroprotective action of DEP in vivo, it deserves a further investigation.

In conclusion, DEP could reduce the moderate neurotoxic effect of $\mathrm{MPP}^{+}$and PQ in rats. These data revealed that DEP might be a useful therapeutic agent in treating the early stage of PD patients.

\section{ACKNOWLEDGMENTS}

We are grateful to Prof. Wang, Y. (Department of Pharmacology, National Defense Medical Center, Taipei, Taiwan, and National Institute on Drug Abuse, Baltimore, MD) and Prof. Hoffer, B.J. (National Institute on Drug Abuse, Baltimore, MD) for their numerous technical support and suggestions. 
This work was supported by grants NSC-882314-B-002-402 and NSC892314-B-385-001 from National Science Council Taipei, Taiwan.

\section{REFERENCES}

Ansari, K. S., Yu, P. H., Kruck, T. P., and Tatton, W. G. (1993). Rescue of axotomized immature rat facial motoneurons by R(-)-deprenyl: Tereospecificity and independence from monoamine oxidase inhibition. J. Neurosci. 13, 4042-4053.

Ballard, P. A., Tetrude, J. W., and Langston, J. W. (1985). Permanent human parkinsonism due to MPTP. Neurology 35, 969-976.

Bagetta, G., Corassniti, T., Iannone, M., Nistico, G., and Stephenson, J. D. (1992). Production of limibic motor seizures and brain damage by systemic and intracerebral injection of paraquat in rats. Pharmacol. Toxicol. 71, 443-448.

Barbeau, A., Roy, M., Cloutier, T., Plasse, L., and Paris, S. (1986). Environ. and genetic factors in the etiology of Parkinson's disease. In Adv. in Neurology (Yahr, M. D., and Bergmann, K. J., Eds.), Vol. 45, pp. 299-306. Raven, New York.

Bayoumi, A. E., Perez-Pertejo, Y., Ordonez, C., Reguera, R. M., Cubri, J. C., Balana-Fouce, R., and Ordonez, D. (2000). Alterations on polyamine content and glutathione metabolism induced by different concentrations of paraquat in CHO-K1 cells. Toxicol. In Vitro 14, 211-217.

Behan, W. M., McDonald, M., Darlington, L. G., and Stone, T. W. (1999). Oxidative stress as a mechanism for quinolinic acid-induced hippocampal damage: Protection by melatonin and deprenyl. Br. J. Pharmacol. 128, 1754-1760.

Bocchetta, A., and Corsini, G.U. (1986). Parkinson's disease and pesticides. Lancet 2, 1163.

Bradbury, A. J., Costall, B., Domeney, A. M., Jenner, P., Kelly, M. E., Marsden, C. D., and Naylor, R. J. (1986). 1-methyl-4-phenylpyridine is neurotoxic to the nigrostriatal dopamine pathway. Nature 319, 56-57.

Brooks, A. I., Chadwick, C. A., Gelbard, H. A., Cory-Slechta, D. A., and Federoff, H. J. (1999). Paraquat elicited neurobehavioral syndrome caused by dopaminergic neuron loss. Brain Res. 823, 1-10.

Burns, R. S., Chiueh, C. C., Markey, S. P., Ebert, M. H., Jacobowitz, D. M., and Kopin, I. J. (1983). A primate model of parkinsonism: Selective destruction of dopaminergic neurons in the pars compacta of the substantia nigra by N-methyl-4-phenyl-1,2,3,6-tetrahydropyridine. Proc. Natl. Acad. Sci. USA 80, 4546-4550.

Bus, J. S., Cagen, S. Z., Olgaard, M., and Gibson, J. E. (1976). A mechanism of paraquat toxicity in mice and rats. Toxicol. Appl. Pharmacol. 35, 501513.

Carlile, G. W., Chalmers-Redman, R. M., Tatton, N. A., Pong, A., Borden, K. E., and Tatton, W. G. (2000). Reduced apoptosis after nerve growth factor and serum withdrawal: Conversion of tetrameric glyceraldehyde-3phosphate dehydrogenase to a dimer. Mol. Pharmacol. 57, 2-12.

Carrillo, M. C., Kanai, S., Nokubo, M., Ivy, G. O., Sano, Y., and Kitani, K. (1992). Deprenyl increases activities of superoxide dismutase and catalase in sriatum but not in hippocampus: The sex and age-related differences in the optimal dose in the rat. Exp. Neurol. 116, 286-294.

Chiueh, C. C., Huang, S. J., and Murphy, D. L. (1992). Enhanced hydroxyl radical generation by 2 '-methyl analog of MPTP: Suppression by clorgyline and deprenyl. Synapse 11, 346-348.

Cohen, G., Pasik, P., Cohen, B., Leist, A., Mytilineou, C., and Yahr, M. D. (1984). Pargyline and deprenyl prevent the neurotoxicity of 1-methyl-4phenyl-1,2,3,6-tetrahydropyridine (MPTP) in monkeys. Eur. J. Pharmacol. 106, 209-210.

Cohen, G., and Spina, M. B. (1989). Deprenyl suppresses the oxidant stress associated with increased dopamine turnover. Ann. Neurol. 26, 689-690.

Creese, I., Burt, D. R., and Snyder, S. H. (1977). Dopamine receptor binding enhancement accompanies lesion-induced behavioural supersensitivity. Science 197, 596-598.

Endo, T., Hara, S., Kuriiwa, F., and Kano, S. (1988). Effects of a paraquatcontaining herbicide, Gramoxon, on the central monoamines and acetylcholine in mice. Res. Commun. Psychol. Psychiatry Behav. 13, 261-270.

Fallon, J., Matthews, R. T., Hyman, B. T., and Beal, M. F. (1997). MPP ${ }^{+}$ produces progressive neuronal degeneration which is mediated by oxidative stress. Exp. Neurol. 144, 193-198.

Finnegan, K. T., Skratt, J. J., Irwin, I., DeLanney, L. E., and Langston, J. W. (1990). Protection against DSP-4-induced neurotoxicity by deprenyl is not related to its inhibition of MAO-B. Eur. J. Pharmacol. 184, 119-126.

Fredriksson, A., Fredriksson, M., and Eriksson, P. (1993). Neonatal exposure to paraquat or MPTP induces permanent changes in striatum dopamine and behavior in adult mice. Toxicol. Appl. Pharmacol. 122, 258-264.

Hertzman, C., Wiens, M., Bowering, D., Snow, B., and Calne, D. (1990). Parkinson's disease: A case-control study of occupational and environmental risk factors. Am. J. Ind. Med. 17, 349-355.

Ho, S. C., Woo, J., and Lee, C. M. (1989). Epidemiologic study of Parkinson's disease in Hong Kong. Neurology 39, 1314-1318.

Hudson, J. L., Levin, D. R., and Hoffer, B. J. (1993). A sixteen-channel automated rotometer system for reliable measurement of turning behavior in 6-hydroxydopamine lesioned and transplanted rats. Cell Transpl. 2, 507514.

Hoffman, A. F., van Horne, C. G., Eken, S., Hoffer, B. J., and Gerhart, G. A. (1997). In vivo microdialysis studies of somatodendritic dopamine release in the rat substantia nigra: Effects of unilateral 6-OHDA lesions and GDNF. Exp. Neurol. 147, 130-141.

Iannone, M., Ciriolo, M. R., Rotilio, G., and Nistico, G. (1991). Intra-nigral infusion of $\mathrm{Cu}$-free superoxide dismutase prevents paraquat-induced behavioral stimulation and EcoG epileptogenic discharges in rats. Neuropharmacology 30, 893-898.

Javitch, J. A., D’Amato, R. J., Strittmatter, S. M., and Snyder, S. H. (1985). Parkinsonism-inducing neurotoxin, N-methyl-4-phenyl-1,2,3,6-tetrahydropyridine: Uptake of the metabolite N-methyl-4-phenylpyridine by dopamine neurons explains selective toxicity. Proc. Natl. Acad. Sci. USA 82, 2173 2177.

Johannessen, J. N., Adams, J. D., Schuller, H. M., Bacon, J. P., and Markey, S. P. (1986). 1-Methyl-4-phenylpyridine (MPP $\left.{ }^{+}\right)$induces oxidative stress in the rodent. Life Sci. 38, 743-749.

Ju, W. Y., Holland, D. P., and Tatton, W. G. (1994). (-)-Deprenyl alters the time course of death of axotomized facial motoneurons and the hypertrophy of neighboring astrocytes in immature rats. Exp. Neurol. 126, 233-246.

Kadiiska, M. B., Hanna, P. M., and Mason, R. P. (1993). In vivo ESR spin trapping evidence for hydroxyl radical-mediated toxicity of paraquat and copper in rats. Toxicol. Appl. Pharmacol. 123, 187-192.

Langston, J. W., Ballard, P., Tetrud, J. W., and Irwin, I. (1983). Chronic parkinsonism in humans due to a product of meperidine analog synthesis. Science 219, 979-980.

Lindquist, N.G., Larsson, B.S., and Lyden-Sokolowski, A. (1988). Autoradiography of $\left[{ }^{14} \mathrm{C}\right]$ paraquat or $\left[{ }^{14} \mathrm{C}\right]$ diquat in frogs and mice: Accumulation in neurimelanin. Neurosci. Lett. 93, 1-6.

Liou, H. H., Chen, R. C., Tsai, Y. F., Chen, W. P., Chang, Y. C., and Tsai, M. C. (1996). Effects of paraquat on the substantia nigra of the Wistar rats: Neurochemical, histological and behavorial studies. Toxicol. Appl. Pharmacol. 137, 34-41.

Liou, H. H., Tsai, M. C., Chen C. J., Jeng, J. S., Chang, Y. C., Chen S. Y., and Chen, R. C. (1997). Environmental risk factors and Parkinson's disease: A case-control study in Taiwan. Neurology 48, 1583-1588.

Lotharius, J. Dugan, L. L., and O'Malley, K. L. (1999). Distinct mechanisms underlie neurotoxin-mediated cell death in cultured dopaminergic neurons. J. Neurosci. 19, 1284-1293. 
Lowry, O. H., Rosebrough, N. J., Farr, A. L., and Randall, R. J. (1951). Protein measurement with the Folin phenol reagent. J. Biol. Chem. 193, 265-275.

Maruyama, W., and Naoi, M. (1999). Neuroprotection by (-)-deprenyl and related compounds. Mech. Ageing Dev. 111, 189-200.

Masek, L., and Richards, R. J. (1990). Interactions between paraquat, endogenous lung amines and antioxidants on isolated mouse Clara cells. Toxicology 63, 315-326.

Melchiorri, D., Reiter, R. J., Sewerynek, E., Hara, M., Chen, L., and Nistico, G. (1996). Paraquat toxicity and oxidative damage. Reduction by melatonin. Biochem. Pharmacol. 51, 1095-1099.

Melchiorri, D., Ortiz, G. G., Reiter, R. J., Sewerynek, E., Daniels, W. M., Pablos, M. I., and Nistico, G. (1998). Melatonin reduces paraquat-induced genotoxicity in mice. Toxicol. Lett. 95, 103-108.

Palkovits, M. (1973). Isolated removal of hypothalamic or other brain nuclei of the brain of the rat. Brain Res. 59, 449-450.

Parkinson Study Group. (1993). Effects of tocopherol and deprenyl on the progression of disability in early Parkinson's disease. N. Engl. J. Med. 328, $176-182$.

Patel, M., Daj, B. J., Crapo, J. D., Fridovich, I., and MacNamara, J. O. (1996). Requirement for superoxide in excitotoxic cell death. Neuron 16, 345-355.

Pellegrini-Giampietro, D. E., Cherici, G., Alesiani, M., Carla, V., and Moroni, F. (1990). Excitatory amino acid release and free radical formation may cooperate in the genesis of ischemia-induced neuronal damage. J. Neurosci. 10, 1035-1041.

Paxinos, G., and Waston, C. (1986). The rat brain in stereotaxic coordinates. Academic Press, London.

Perlow, M. J., Fread, W. J., Hoffer, B. J., Seiger, A., Olson, L., and Wyatt, R. J. (1979). Brain grafts reduce motor abnormalities produced by destruction of nigrostriatal dopamine system. Science 204, 643-647.

Sanchez-Ramos, J. R., Hefti, F., and Weiner, W. J. (1987). Paraquat and Parkinson's disease. Neurology 37, 728.

Salonen, T., Haapalinna, A., Heinonen, E., Suhonen, J., and Hervonen, A. (1996). Monoamine oxidase B inhibitor selegiline protects young and aged rat peripheral sympathetic neurons against 6-hydroxydopamine-induced neurotoxicity. Acta Neuropathol. 91, 466-474.

Semkov, I., Wolz, P., Schilling, M., and Krieglistein, J. (1996). Selegilline enhances NGF synthesis and protects central nervous system neurons from excitotoxic and ischemic damage. Eur. J. Pharmacol. 315, 19-30.

Seniuk, N. A., Henderson, J. T., Tatton, W. G., and Roder, J. C. (1994). Increased CTNF gene expression I process-bearing astrocytes following injury is augmented by R(-)-deprenyl. J. Neurosci. Res. 37, 278-286.

Seto, Y., and Shinohara, T. (1988). Structure-activity relationship of reversible cholinesterase inhibitors including paraquat. Arch. Toxicol. 62, 37-40.

Sharp, C. W., Ottolenghi, A., Posner, H. S. (1972). Correlation of paraquat toxicity with tissue concentrations and weight loss of the rat. Toxicol. Appl. Toxicol. 22, 241-251.

Singh, Y., Swanson, E., Sokoloski, E., Kutty, R. K., and Krishna, G. (1988). MPTP and MPTP analogs induced cell death in cultured rat hepatocytes involving the formation of pyridinium metabolites. Toxicol. Appl. Pharmacol. 96, 347-359.

Sirinathsinghji, D. J. S., Heavens, R. P., Richards, S. J., Beresford, I. J. M., and Hall, M. D. (1988). Experimental hemiparkinsonism in the rat following chronic unilateral infusion of $\mathrm{MPP}^{+}$into the nigrostriatal dopamine path- way-1. Behavioral, neurochemical and histological characterization of the lesion. Neuroscience 27, 117-128.

Sun, C. J., Johannessen, J. N., Gessner, W., Namura, I., Singhaniyom, W., Brossi, A., and Chiueh, C. C. (1988). Neurotoxic damage to the nigrostriatal system in rats following intranigral administration of MPDP + and $\mathrm{MPP}^{+}$. J. Neural. Transm. 74, 75-86.

Tang, Y. P., Ma, Y. L., Chao, C. C., Chen, K. Y., and Lee, E. H. (1998). Enhanced glial cell line-derived neurotrophic factor mRNA expression upon (-)-deprenyl and melatonin treatments. J. Neurosci. Res. 53, 593-604.

Tanner, C. M. (1989). The role of environmental toxins in the etiology of Parkinson's disease. Trends Neurosci. 12, 49-54.

Tanner, C. M., and Goldman, S. M. (1996). Epidemiology of Parkinson's disease. Neuroepidemiology 14, 317-335.

Tatton, W. G., Ju, W. Y., Holland, D. P., Tai, C., and Kwan, M. (1994). (-)-Deprenyl reduces PC12 cell apoptosis by inducing new protein synthesis. J. Neurochem. 63, 1572-1575.

Thomas, C. E., Huber, E. W., and Ohlweiler, D. F. (1997). Hydroxyl and peroxyl radical traping by the monoamine-B inhibitors deprenyl and MDL 72,974A: Implications for protection of biological substrates. Free Radical Biol. Med. 22, 733-737.

ThyagaRajan, S., Stevens, S. Y., and Felten, D. L. (1999). Region-specific alterations in the concentrations of catecholamines and indoleamines in the brains of young and old F344 rats after L-deprenyl treatment. Brain Res. Bull. 48, 513-520.

Trush, M. A., Mimnaugh, E. G., Ginsberg, E., and Gram, T. E. (1981). In vivo stimulation by paraquat of reactive oxygen-mediated lipid peroxidation in rat lung microsomes. Toxicol. Appl. Pharmacol. 60, 279-286.

Tsai, Y. F., Tsai, H. W., and Tai, M. Y. (1994). Comparison of brain dopamine depletion induced by low-dose 1-methyl-4-phenyl-1,2,3,6-tetrahydropyridine (MPTP) in young and aged rats. Neurosci. Res. 20, 27-33.

Ungerstedt, U., and Arbuthnott, F. W. (1970). Quantitative recording of rotational behavior in rats after 6-hydroxydopamine lesions of the nigrostriatal dopamine system. Brain Res. 24, 485-493.

Wadia, J. S., Chalmers-Redman, R. M. E., Ju, W. J. H., Carlile, G. W., Phillips, J. L., Fraser, A. D., and Tatton, W. G. (1998). Mitochondrial membrane potential and nuclear changes in apoptosis caused by serum and nerve growth factor withdrawal: Time course and modification by (-)-deprenyl. J. Neurosci. 18, 932-947.

Wang, Y., Lin, J. C., Chiou, A. L., Lin, J. Y., Liu, J. C., and Zhou, F. C. (1995). Human ventromesencephalic graft restore dopamine release and clearance in hemiparkinsonisn rats. Exp. Neurol. 136, 98-106.

Wu, R. M., Chiueh, C. C. Pert, A., and Murphy, D. L. (1993). Apparent antioxidant effect of 1-deprenyl on hydroxyl radical formation and nigral injury elicited by $\mathrm{MPP}^{+}$in vivo. Eur. J. Pharmacol. 243, 241-247.

Wu, R. M., Murphy, D. L., and Chiueh, C. C. (1996). Suppression of hydroxyl radical formation and protection of nigral neurons by $l$-deprenyl (selegiline). Ann. NY Acad. Sci. 786, 379-390.

Yang, W. L., and Sun, A. Y. (1998). Paraquat-induced cell death in PC12 cells. Neurochem. Res. 23, 1387-1394.

Zsilla, G., Foldi, P., Held, G., Szekely, A. M., and Knoll, J. (1986). The effect of repeated doses of (-)-deprenyl on the dynamics of monoaminergic transmission: Comparison with clorgyline. Pol. J. Pharmacol. Pharm. 38, $57-67$. 